Teaching Tips (COVID)

\title{
The Do-It-Yourself Electrocardiogram
}

\author{
Eileen HaAse $10,{ }^{1}$ Ryan O’Hara, ${ }^{1}$ and Anil Maybhate ${ }^{2}$ \\ ${ }^{1}$ Department of Biomedical Engineering, Johns Hopkins University, Baltimore, MD 21218, USA; and ${ }^{2}$ Applied Biomedical \\ Engineering - Engineering for Professionals, Johns Hopkins University, Baltimore, MD 21218, USA
}

(Received 11 April 2021; accepted 9 December 2021; published online 7 February 2022)

\begin{abstract}
Hands-on labs are a critical component of biomedical engineering undergraduate education. Due to both the pandemic and the growing interest in online education, we developed a Do-it-yourself Electrocardiogram (DIY EKG) project. The Arduino-based DIY EKG kit instructed students how to build a circuit to obtain their own EKG and then analyze their EKG data using Matlab. Despite the obstacles of virtually trouble-shooting, $85.4 \%$ of students $(n=103)$ were able to obtain their own EKG at home. We have provided the labelled circuit drawings, stepby-step instructions, Matlab files, and results in this paper. Survey results indicate that $89 \%$ of students felt the DIY EKG project was a "challenging yet fulfilling experience."
\end{abstract}

Keywords-Online teaching, Experiential learning, Electrocardiogram, Arduino, Remote learning.

\section{CHALLENGE STATEMENT}

One of the important skills for biomedical engineering students is the ability to acquire and analyze data from living tissue. The SARS-COV-2 pandemic in 2019-2021 limited student access to a hands-on upper-level undergraduate cardiology lab, where students had previously recorded from beating frog hearts and used electrodes to record their own electrocardiograms (EKGs). Our challenge was to provide a mechanism for students to learn cardiac electrophysiology and mechanics through a combination of virtual and remote hands-on activities.

This half-semester one-credit lab was designed to supplement a lecture course focused on mathematical

Address correspondence to Eileen Haase, Department of Biomedical Engineering, Johns Hopkins University, Baltimore, MD 21218, USA. Electronic mail: Ehaase1@jhu.edu

Eileen Haase, Ryan O'Hara, and Anil Maybhate have contributed equally to this work. models of the electrophysiology and mechanics of the heart. The cardiovascular system (CVS) is incredibly complex. While models of the CVS provide a useful mechanism for students to manipulate one variable at a time, obtaining their own EKG truly brings the equations to life. A hybrid teaching situation, in which students combine mathematical models with hands-on physiology, has been shown to provide a solid foundation in understanding the CVS. ${ }^{1}$ We wanted to make sure that students obtained the same educational value as an in-person lab in which they obtain and analyze cardiac data from living tissue; in this case, their own hearts. Another goal of the lab was for students to engage and learn from each other during their weekly sessions. Active, peer-based, learning is a proven evidence-based teaching practice. ${ }^{2-4}$ Ensuring students worked with each other to build a circuit and obtain their own EKG maintained the interactive and collegial aspects of the course even though it was taught entirely online.

\section{NOVEL INITIATIVE: DO-IT-YOURSELF ELECTROCARDIOGRAM (DIY EKG) PROJECT}

Since students could no longer come into lab to obtain and analyze data, we developed a combination of virtual and at-home labs. Students analyzed Biopac ${ }^{5}$ sample data or de-identified patient data. In addition, we mailed each student a send-home Arduino-based kit which provided the materials needed to build their own circuit and obtain their own electrocardiogram - the Do-It-Yourself EKG (DIY EKG). We are not the first to develop a "lab-in-the box"., 6,7 There 
have also been other portable and student-built circuits to measure EKGs. ${ }^{8-11}$ Recent work has demonstrated the possibility of a low-cost Arduino-based EKG using Bluetooth, which could be beneficial in underdeveloped communities. ${ }^{12}$ Our initiative allows students to analyze and graph EKG data on their own computers using Matlab. Safety is a concern with all student-built circuits. Every student completed the university's online courses in Lab Safety and Human Subjects Research prior to the start of the semester, and were reminded that obtaining their own EKG data was completely voluntary. We individually viewed each student's circuit (virtually) to avoid errors in the set-up and the very real possibility of electrical shock if the circuit was not built and used correctly. ${ }^{13-16}$

\section{VIRTUAL LABS}

Students $(n=103)$ met weekly, for four hours, on Microsoft (MS) Teams, to work with their lab partner on four different cardiac labs:

(1) Introduction to EKGs-Analyzing normal EKG data.

(2) EKGs in the clinic-Analyzing patient EKG data.

TABLE 1. Lab course objectives.

Lab Objectives for in-person and online lab course

1. Formulate hypothesis for experiments, including those on living systems

2. Devise procedures for experiments, including those on living systems

3. Collect and validate data using appropriate equipment and data acquisition systems

4. Display, describe, summarize and interpret experimental results in a lab report

5. Compare the experimental results to previous work and computational models

6. Understand the guidelines for ethical use of animals/humans in research

7. Develop laboratory skills applied to living systems

TABLE 2. Lab topics and learning objectives.

Topic

Introduction to

Electrocardiograms

EKGs in the clinic

Do-it-yourself
Electrocardiogram
(DIY EKG)

Cardiac Catheterization
Learning objectives

- Analyze an ECG from Leads I and III and identify the primary waves (P, QRS, T)

- Identify the factors that cause changes in the ECG, both from one person to another, and for one person at different periods of time.

- Compare the displayed calculated ECG Lead II to recorded ECG Leads I and III, and use the R-wave amplitudes to confirm Einthoven's Law

- Approximate the mean electrical axis of the ventricles on the frontal plane using vectors derived from the amplitude and polarity of the QRS complex in Leads I and III.

- Sketch a Wigger's diagram and pressure-volume loop using your ECG, arterial blood pressure, and estimates of total blood volume

- List the steps required to conduct a 12-lead EKG using clinical equipment

- Explain how to set-up a continuous monitoring EKG system using clinical equipment

- Describe the main clinical indicators for a patient to receive an EKG in the ER

- Explain what an EKG can diagnose and the limitations

- Identify common EKG abnormalities

- Build, test, trouble-shoot and demonstrate a simple EKG amplifier on a breadboard

- Interface, program and control a microcontroller board (Arduino) with your computer

- Monitor, acquire, store and analyze a digitized single lead EKG signal using the Arduino board and directly using MATLAB.

- Identify prominent waves (PQRST) in the human EKG

- Determine the heart rate and other simple parameters from a single lead EKG signal

- Compare the various imaging modalities used in the cardiac catheterization lab, how they are used, and their limitations in the lab

- Analyze hemodynamic data and anatomic measurements collected during catheterization lab procedures

- Analyze the frequency response of a pressure catheter

- Compare pressure waveforms acquired from different locations in the human body

- Compute the approximate aortic valve area assuming Bernoulli's equation applies to flow (Gorlin Equation) 
(3) DIY EKG project-Building a circuit to obtain and analyze student's own data.

(4) Cardiac Catheterization-Analyzing cardiac catheterization patient data.

Each of the four labs used pre-recorded videos and handouts to provide background information. The three labs which analyzed sample or patient data had a multiple-choice pre-lab quiz, based on the videos, to ensure that students were prepared for each lab. The DIY EKG project had a pre-lab assignment instead of a quiz. Sample pre-lab quiz questions and the DIY EKG pre- and post-lab assignment are included in Appendix 1. Students used the four-hour lab time each week to work in pairs on the lab reports. However, each student was mailed their own EKG kit and required to build their own circuit. The course objectives were met through the combination of virtual and kit-based labs. Table 1 lists the lab course objectives, which align with the Accreditation Board for Engineering and Technology (ABET) student outcomes. ${ }^{17}$ Table 2 summarizes the specific learning objectives for each of the four labs.

The first lab used Biopac Student Data to evaluate normal EKGs, identify the PQRST waves, and apply Einthoven's Law to calculate lead values. The PQRST waves refer to the changes in voltage due to atrial depolarization $(\mathrm{P})$, ventricular depolarization $(\mathrm{QRS})$ and ventricular repolarization $(\mathrm{T})$ of the heart. The second lab focused on abnormal patient EKGs, including atrial and ventricular fibrillation, bradycardia, and tachycardia. The fourth lab focused on information gleaned from cardiac catheterization and how this technique could be used to replace a heart valve (Transcatheter Aortic Valve Replacement-TAVR) or insert a stent. The three virtual labs were supplemented with live lectures from clinicians during which students could ask questions during class.

\section{DIY EKG LAB}

We developed and mailed an Arduino-based DIY EKG kit to each student at home, with addresses from around the world. While this was quite an expense for the biomedical engineering (BME) department, it was the only way to ensure equity for our students. We wanted each student to receive the same kit, on time, with all the needed components. We were concerned some students might not be able to afford the kit or might be missing key parts if they ordered everything

\footnotetext{
*Note: students completed online training in Human Subjects Research and were explicitly advised not to use their personal EKG data for any medical interpretations.
}

themselves. ${ }^{18}$ To make the DIY EKG project easier for faculty to replicate in their own courses, we have included the list of parts for the kits in Table 3, a labelled picture of the DIY EKG circuit (Fig. 1) and a schematic diagram (Fig. 2). The students used Matlab to analyze and graph the data. We have included a link to a YouTube video (https://www.youtube.com/watch?v $=$ rkYqcUv10Zs) with step-by-step instructions for building the EKG circuit and obtaining your own EKG data, as well as detailed written instructions and follow-up questions in Appendix II. The Matlab script files are in Appendix III. The Arduino was programmed using resources available on the Sparkfun website. ${ }^{19}$

Our EKG circuit includes a standard instrumentation amplifier (INA 128) with a simple low-pass RC filter and a high-pass RC filter, both designed using the standard LM741 ICs. The filters can be connected in sequence without a DC offset since we are using a simple DC battery power as the power source, which introduces insignificant DC shifts. The two filters combined act as a band-pass filter. Consequently, the final signal acquired was free of high-frequency noise and/or any low-frequency bio-noise coming from breathing or movement artifacts. Barring these artifacts, the EKG is the biggest electrophysiological signal generated by human body and as such we expect the acquisition to be fairly simple and robust. The standard clinical EKG is typically acquired and analyzed within the $0.5-100 \mathrm{~Hz}$ frequency bands. Our circuit component values were selected to achieve as close a result as possible, with the constraint of commercially readily available electronic components that

TABLE 3. Components and tools for DIY EKG Amplifier.

\begin{tabular}{lll}
\hline Part \# & \multicolumn{1}{c}{ Description } & Quantity/Kit \\
\hline 1 & Digital Multimeter with 9V Battery & 1 \\
2 & Wire Stripper & 1 \\
3 & Needle Nose Pliers & 1 \\
4 & Breadboard-Classic & 1 \\
5 & 9V Alkaline Battery & 4 \\
6 & 9V Snap Connector & 3 \\
7 & Jumper Wire Kit-140pcs & 1 \\
8 & Jumper Wires Premium 6“ M/M Ribbon & 1 \\
9 & Arduino Chip-SparkFun Leonardo Qwicc & 1 \\
10 & USB mini-B Cable & 1 \\
11 & Sensor Cable Electrode Pads 3pk & 2 \\
12 & Sensor Pads 10pk & 3 \\
13 & Audio Jack 3.5mm & 2 \\
14 & Polarized Connectors-Header (2-pin) & 4 \\
15 & Resistor Kit -1/4W (500 total) & 1 \\
16 & 3.3 Mega Ohm Resistors & 5 \\
17 & Capacitor Kit & 1 \\
18 & LM741 OpAmp Ics & 4 \\
19 & INA128 Instrumentation Amplifier Ics & 2 \\
\hline
\end{tabular}


could be easily purchased by a student, if needed, from a local electronic hardware outlet.

The functionality of the circuit-including the instrumentation amplifier and the two filters-was explained to the students in detail using the online asynchronous videos included with the lab module. The students were quizzed on specifics of this functionality before they started to build the circuit. The

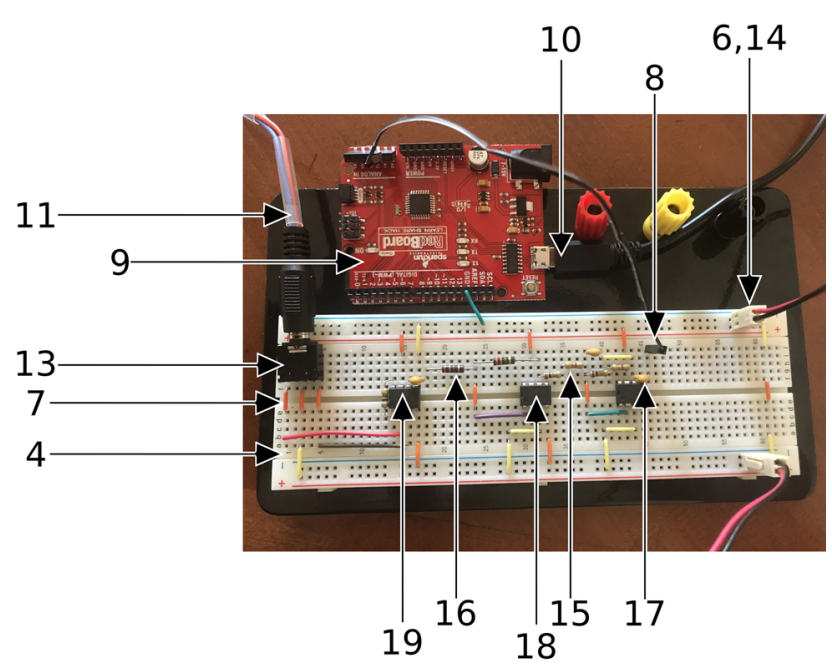

FIGURE 1. DIY EKG circuit: The numbers represent the kit components listed in Table 3.
DIY EKG prelab and postlab assignments are supplied in Appendix I.

\section{SAFETY CONCERNS}

The video lectures in the online component of the module detail the knowledge about circuit function and safety. Prior to connecting each circuit and acquiring the signal from an individual student's body surface, the instructors and TAs inspected the circuit in every detail to ensure that the component values and the connections were secure and safe. The USB connection to the computer is only one way - for data acquisition-from the SPARKFUN board to the computer. There is almost no current flowing back from the computer to the circuit that could lead back to the human body. We have assumed that small voltages are not dangerous as long as the impedance to the body is high enough to prevent large currents, specifically currents less than 5 milliamps. ${ }^{13-16}$ Wet skin could reduce the impedance such that a $9 \mathrm{~V}$ battery combined with an improperly built circuit could cause a shock. Attaching the circuit to an electrical outlet, instead of just the battery, is not advised. The circuit as designed, with battery powered Op-Amps, has current flow of the order of a few hundred microvolts, which is orders of magnitudes lower than even the few milli-

\section{EKG Amplifier Circuit Using INA128 (without DC Offsets) but with modifications for the kit implementation}

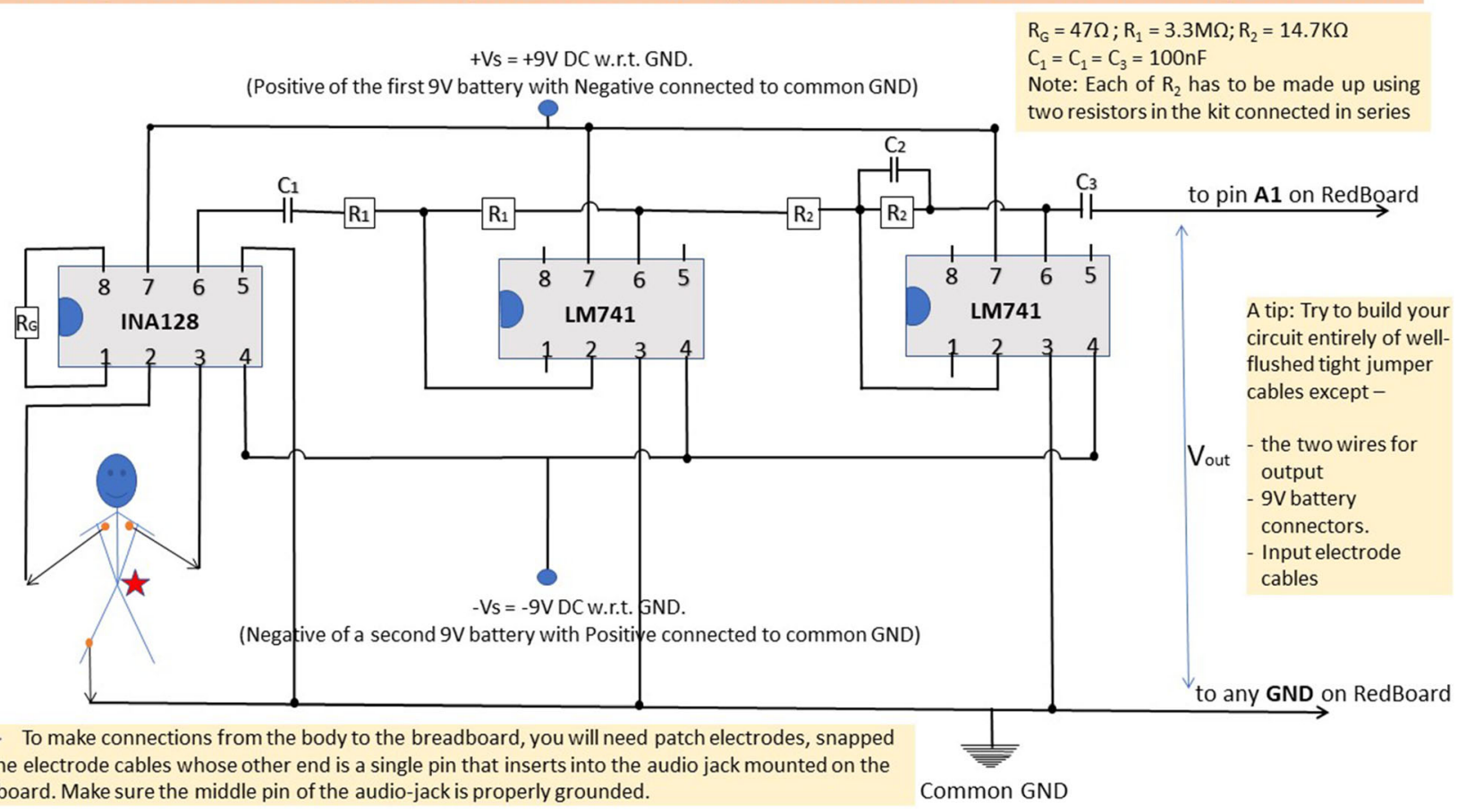

FIGURE 2. EKG Amplifier Circuit Schematic. See Appendix II for step-by-step instructions on how to build the circuit. 
amps level that could cause a perceptible shock in humans. $^{11}$

The students were instructed to check and verify the voltages with a digital multimeter before connecting themselves to the circuit. Students should be told to only use battery power for their computers while obtaining data. Thank you to the reviewers who pointed out the need for safety measures beyond what we have already implemented. This has motivated us to look into our design. In the future, we will explore optical isolation between the instrumentation amplifier stage and the electrode leads that are connected to the skin.

All safety checks should be performed in front of the TA/faculty and be a required part of the project grade. In addition to the important safety concerns, students should be reminded that any home-made device is not approved for medical use. ${ }^{20}$

My work in this lab has given me a solid background in understanding EKGs and cardiac catherization (I will remember the key points a year from now)

The pre-lab quizzes helped me to prepare for the lab sessions with my classmates.

The "Analyzing EKG Data" module using sample Biopac data helped me to understand the details of the EKG and provided a solid background for the rest of the course.

"EKGs in the clinic" and the lecture by Dr. X provided clinical relevance which was a worthwhile and productive learning experience.

The "DIY EKG" using send-home Arduino kits to build a circuit and obtain and analyze my own EKG data was a challenging yet fulfilling learning experience

The "Cardiac Catheterization" module and the lecture by $\mathrm{Dr}$. $\mathrm{Y}$ helped me to understand how and why catheters are used in the clinic.

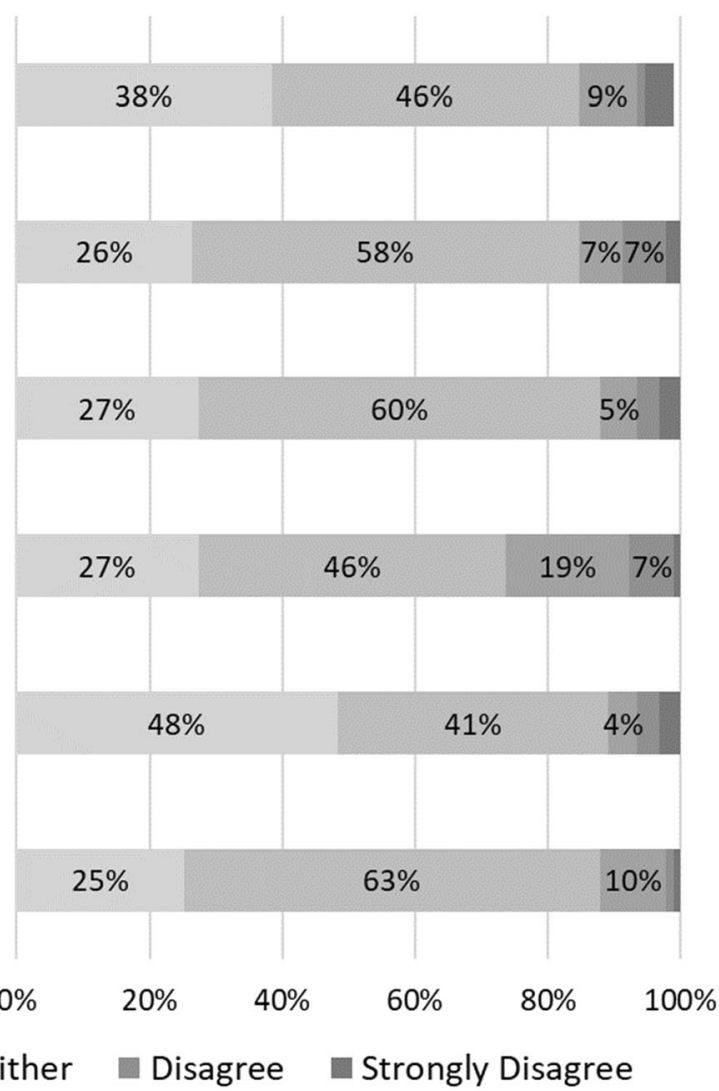

FIGURE 3. Anonymous Survey Data on student perceptions of the four modules (data values of less than $5 \%$ are not shown for clarity). $\mathbf{8 4} \%$ of students agreed that they would remember the key points of the four labs in a year. Three-quarters of the class felt all four labs were "productive learning experiences", with the DIY EKG lab receiving the highest ratings (89\%).
Transitioning an in-person, animal-based, physiology lab course online, especially when given little notice, is challenging under the best of circumstances. We wanted students to understand cardiac physiology at a much deeper level than a lecture could provide. We required students to prepare for each lab in advance by viewing the background material and passing the prelab quiz/assignment. Survey data indicated that $84 \%$ of students $(n=103)$ agreed or strongly agreed that "The pre-lab quizzes helped me to prepare for the lab sessions with my classmates." This preparation ensured that students were ready to collaborate with their classmates to learn as much as possible during the weekly four-hour lab sessions on MS Teams. Students analyzed both normal and patient data, which not only gave a clinical perspective, but also emphasized the critical features of the waveforms for both the EKG and aortic flow data.

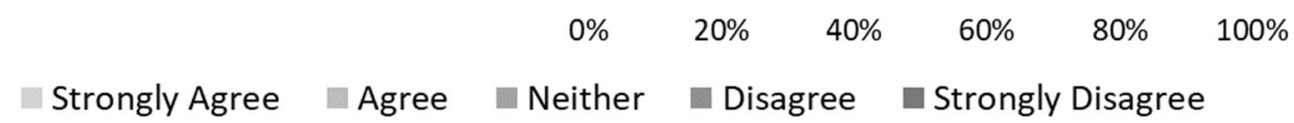




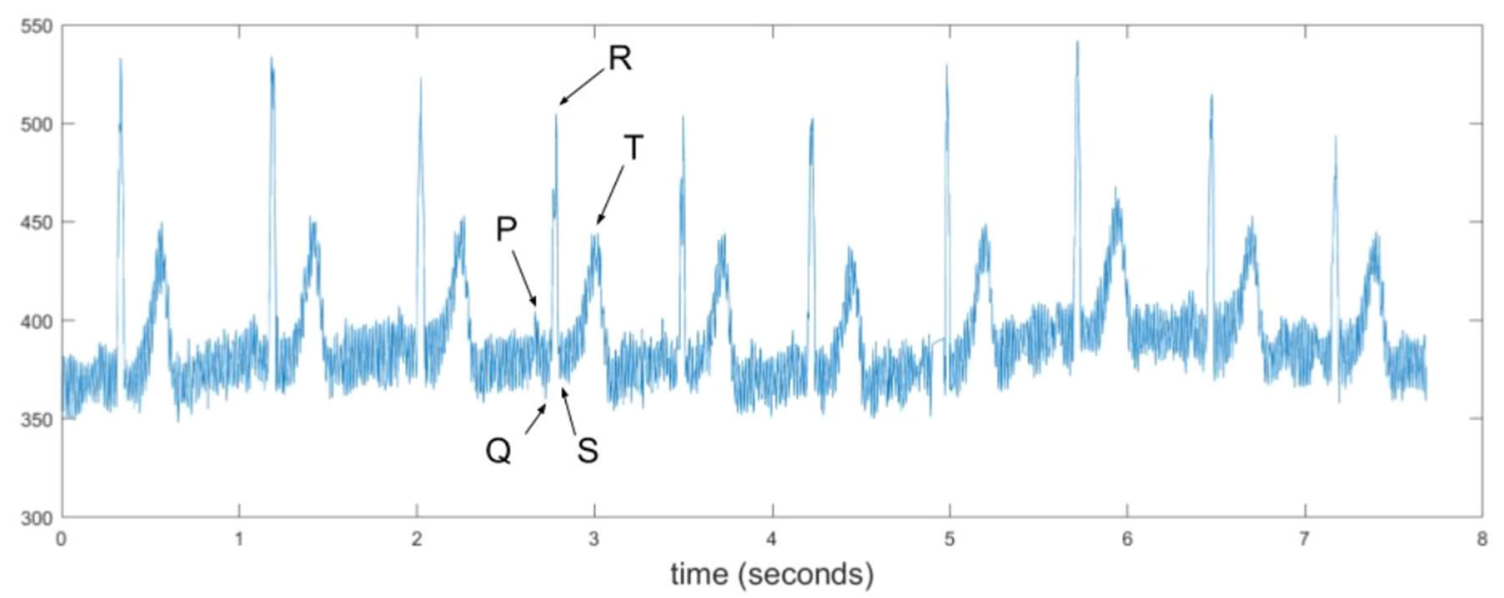

FIGURE 4. Sample single-lead EKG tracing with the P (atrial depolarization), QRS (ventricular depolarization) and T (ventricular repolarization) waves labelled. Note that the heart rate in this sample can be calculated from the $\mathbf{R}-\mathbf{R}$ distance, which is approximately four heartbeats in three seconds or $\mathbf{8 0}$ beats per minute.

TABLE 4. Student comments on the DIY EKG project.

I thought the do it yourself EKG lab was incredibly cool. It was the only hands-on thing l've been able to do since COVID started so it was really refreshing/fun as well as being a great learning experience.

ECG lab was a lot of fun! I loved it, I loved building it, I definitely learned a lot during the lab.

I liked the DIY EKG kits. (The TA's) instructions were easy to follow, and it was fun to build. It was cool to see my own EKG reading.

The DIY EKG was one of the my favorite parts of the class, and I got a lot of satisfaction from being able to actually build something instead of just using a lab machine to measure my EKG

I think the EKG kits allowed students (at least me) to enjoy an aspect of the course that felt more in-person.

I loved the DIY EKG lab. Thank you so much for mailing those kits out!

DIY EKG was a great experience and the highlight of the course. I've never worked with a breadboard or Arduino before and now I'm excited to try out more hardware-y / EE type stuff.

I think the building of EKG is the best project/module among all, it's a lot of fun building it and it's so cool to collect my own data. Isn't it just so much fun to brag about how you now have an EKG device at your home?

my ekg didn't work after $\sim 4$ hours of troubleshooting and l'm not sure why :(

Measuring my own ECG was a great experience.

Building the EKG was incredibly fun (at times frustrating) and truly made me feel like an engineer!

BUILDING THE EKG WAS THE HIGHLIGHT OF MY SEMESTER!!

Building the arduino EKG was a great way to apply what I had learned and gain hands on experience with EKG technology.

The building an EKG lab was my favorite because it was the first time I was able to apply all the electrical engineering knowledge we have been taught at a theoretical level.

The best part (of the course) was building an EKG and taking data on myself. Especially in the remote learning environment, this felt much more like an in-person part of the course.

The DIY EKG was really cool, and I was so amazed to see my EKG and to have been able to record it myself! It was definitely not too easy troubleshooting, but I got a lot of help, and in the end the time I spent on it was worth it.

This was the first bme class where I could see how what we learned in class could be applied in practice out of the classroom Analyzing my own EKG readings that I took w/ my breadboard + arduino setup is one of the coolest things I've ever done.

The end-of-semester anonymous survey data for each of the four modules indicated that $84 \%$ of students felt they gained a solid understanding of EKGs and cardiac catherization, and would remember the key points a year from now (Fig. 3). Almost half of the students strongly agreed $(48 \%)$ or agreed $(41 \%)$, a total of $89 \%$, that "The DIY EKG using send-home Arduino kits to build a circuit and obtain and analyze my own EKG data was a challenging yet fulfilling learning experience". Troubleshooting virtually had challenges.
A few kits had broken pins or the wrong components, which added another layer of complexity to the project. In many cases, this issue was resolved by stripping the stranded wires and inserting them directly into the breadboard.

Despite the obstacles, $85.4 \%(88 / 103)$ of students were able to obtain their own EKG at home. Students who could not get a working signal after spending time troubleshooting were allowed to use data from another student. A sample single-lead EKG tracing is shown in 
Fig. 4. Note that the EKG recording in Fig. 4 clearly depicts the PQRST waves and can be used to calculate the heart rate by measuring the time between R-waves ( $\mathrm{R}-\mathrm{R}$ delta time). The combination of virtual and remote labs allowed students to develop expertise in recognizing the components of a normal EKG, specifically the PQRST waves, prior to obtaining their own real-time measurements.

Student comments about the project are listed in Table 4. The comments made it evident that the students appreciated the hands-on experience and teamwork involved in obtaining their own EKGs. The project made students "feel like an engineer", made the course feel "more in-person", and for at least one student was "THE HIGHLIGHT OF MY SEMESTER!!"

Expecting students to analyze EKGs and catheterization data remotely required clear background videos and instructions. The survey data indicated that $71 \%$ of students agreed "The posted online videos were clear to understand and provided the background information needed to complete the assignments" and 86\% felt they were "were interesting and professionally done."

We used MS Teams for the four-hour lab sessions to foster a sense of community and provide ample access to faculty and TAs, since peer-instruction is a critical component of learning. ${ }^{4}$ While two-thirds of the students felt "The Thursday and Friday MS Teams "Groups" allowed me to work well with my classmates and get my lab reports completed", 17\% disagreed, often due to connectivity issues. The advantage of MS Teams was the all-day online access to the TAs, in which $88 \%$ agreed "The MS Teams "TA" Channels allowed me to ask a TA questions and get assistance from 9:00 to 5:30 on Thursday and Fridays." Very few students, less than $2 \%$, used MS Teams frequently outside of the lab. A few representative student comments on MS Teams are below:

- Teams worked surprisingly well for the course. In the past I have hated it for other classes and events, however it was well suited to this class and made sense to use.

- MS Teams is a bit more difficult to navigate than zoom but it provided a good platform for this class

- I loved that the TAs were available through the duration of the class. It made completing the labs during one lab session very attainable.

- Being able to stop by the TAs' meetings were very helpful

- I thought for the TA sessions, teams was a good idea! For meeting $w /$ my lab partner/classmate, I personally preferred using zoom.
Another advantage of MS Teams is that it provided a platform for students to post questions 24/7. Review sessions and office hours were held on MS Teams and could be recorded. We have continued to use MS Teams for both office hours and posting/answering questions this semester, even though we are teaching the lab in-person and have TAs available all-day Thursday and Friday.

In previous years, students obtained their own EKG data using the Biopac Data Acquisition System. Due to the pandemic, students built an Arduino-based circuit to obtain their own EKG data. Consequently, in addition to learning about cardiac electrophysiology, students developed trouble-shooting skills and an understanding of the different types of measurement error. Our primary concern with students building a circuit alone at home, and connecting it to their chest through low impedance electrodes, is that it can pose a safety risk if not done properly. While the risk is small, and the potential learning opportunity is significant, the utmost care must be taken to ensure that the circuit is built and used correctly.

The success of the DIY EKG project has led us to re-evaluate future elements of this lab course. Obtaining and analyzing experimental data from living tissue, such as a frog heart, has always been a component of understanding the electrophysiology and mechanics of the heart. While online labs can be as effective as in-person labs ${ }^{21}$ and offer opportunities for remote learning for students with disabilities or cost concerns, ${ }^{22}$ it is important to consider the comradery of in-person labs. Incorporating an instrumentation project within this cardiac lab gives students the opportunity to build their own equipment to obtain cardiac data and practice trouble-shooting. Based on the students' comments, the time spent was worth the effort. Future in-person offerings of this course will consider including an instrumentation component.

\section{SUPPLEMENTARY INFORMATION}

The online version of this article contains supplementary material available https://doi.org/10.1007/s43 683-021-00061-0.

\section{FUNDING}

Not applicable.

\section{AUTHOR CONTRIBUTIONS}

All three authors contributed equally to this publication. 


\section{CONFLICT OF INTEREST}

Not applicable.

\section{CONSENT TO PARTICIPATE}

Not applicable.

\section{CONSENT FOR PUBLICATION}

Received IRB approval to publish anonymous survey data and overall class results.

\section{ETHICAL APPROVAL}

Not applicable.

\section{REFERENCES}

${ }^{1}$ Christ, A., D. Barowsky, M. Gekle, and O. Thews. A hydraulic model of cardiovascular physiology and pathophysiology embedded into a computer-based teaching system for student training in laboratory courses. $A d v$ Physiol Educ 44(3):423-429. https://doi.org/10.1152/advan. 00069.2020.

${ }^{2}$ Prince, M. Does active learning work? A review of the research. J Eng Educ 93(3):223-231.

${ }^{3}$ Freeman, S., S. L. Eddy, M. McDonough, M. K. Smith, N. Okoroafor, H. Jordt, and M. P. Wenderoth. Active learning increases student performance in science, engineering, and mathematics. Proc Natl Acad Sci USA 111(23):8410-8415.

${ }^{4}$ Crouch, C. H., and E. Mazur. Peer instruction: ten years of experience and results. Am J Phys 69(9):970-977.

${ }^{5}$ Biopac (https://www.biopac.com/).

${ }^{6}$ Mujica FA, Esposito WJ, Gonzalez A, Qi CR, Vassos C, Wieman M, Wilcox R, Kovacs GT, Schafer RW. Teaching digital signal processing with Stanford's Lab-in-a-Box. In 2015 IEEE Signal Processing and Signal Processing Education Workshop (SP/SPE) 2015 Aug 9 (pp. 307-312). IEEE.

${ }^{7}$ Novo, M., N. Sánchez, M. Gutiérrez López, R. G. Cánovas, F. Pardos, D. Trigo, and C. D. Díaz. The lab in a box: a take-out practical experience for an online invertebrate biology course. Invertebr Biol 140(1):e12324. https://doi.o $\mathrm{rg} / 10.1111 /$ ivb. 12324 .

${ }^{8}$ Natumploy S, Sophrom R, Bunnjaweht, D. Portable ECG Display: An Experiential Learning through a Senior De- sign Project. In: 2018 11th Biomedical Engineering International Conference (BMEiCON) (2018), pp. 1-4. https://d oi.org/10.1109/BMEiCON.2018.8609964.

${ }^{9}$ Thanawattano, C., R. Pongthornseri, and S. Dumnin. Wearable wireless ECG sensor with cross-platform realtime monitoring. IEEE-EMBS Conference on Biomedical Engineering and Sciences 2012:284-287. https://doi.org/10. 1109/IECBES.2012.6498035.

${ }^{10}$ DIY ECG EKG Portable Heart Monitor, https://www.ins tructables.com/DIY-ECG-EKG-Portable-Heart-Monitor/.

${ }^{11}$ ECG Display with Arduino, https://www.instructables.co m/ECG-Display-With-Arduino/.

${ }^{12}$ Iskandar WJ, Roihan I, Koestoer RA. Prototype low-cost portable electrocardiogram (ECG) based on Arduino-Uno with Bluetooth feature. In AIP Conference Proceedings 2019 Dec 10 (Vol. 2193, No. 1, p. 050019). AIP Publishing LLC.

${ }^{13}$ Fish, R. M., and L. A. Geddes. Conduction of electrical current to and through the human body: a review. Eplasty 9:e44.

${ }^{14}$ Dalziel Charles, F., and W. R. Lee. Reevaluation of lethal electric currents. IEEE Trans Indus Gen Appl 4(5):467-476. https://doi.org/10.1109/TIGA.1968.4180929.

${ }^{15}$ United States Department of Labor Occupational Health and Safety Administration, https://www.osha.gov/sites/def ault/files/2019-04/Basic_Electricity_Materials.pdf.

${ }^{16}$ Kono M, Takahashi T, Nakamura H, Miyaki T, Rekimoto J. Design Guideline for developing safe systems that apply electricity to the human body. ACM Trans Comput-Hum Interact. 25:3. Article 19. (2018). https://doi.org/10.1145/ 3184743

${ }^{17}$ Accreditation Board for Engineering and Technology (ABET) https://www.abet.org/.

${ }^{18}$ Theobald, E. J., M. J. Hill, E. Tran, S. Agrawal, E. N. Arroyo, S. Behling, N. Chambwe, D. L. Cintrón, J. D. Cooper, G. Dunster, and J. A. Grummer. Active learning narrows achievement gaps for underrepresented students in undergraduate science, technology, engineering, and math. Proc Natl Acad Sci USA 117(12):6476-6483.

${ }^{19}$ Sparkfun (https://learn.sparkfun.com/tutorials/redboard-h ookup-guide).

${ }^{20}$ Feldman, M. D., A. J. Petersen, L. S. Karliner, and J. A. Tice. Who is responsible for evaluating the safety and effectiveness of medical devices? The role of independent technology assessment. J Gen Int Med 23:57-63. https://d oi.org/10.1007/s11606-007-0275-4.

${ }^{21}$ Reuter, R. Online versus in the classroom: student success in a hands-on lab class. Am Distance Educ 23(3):151-162.

${ }^{22}$ Heradio, R., L. de la Torre, D. Galan, F. J. Cabrerizo, E. Herrera-Viedma, and S. Dormido. Virtual and remote labs in education: a bibliometric analysis. Comput Educ 98:1438. 